\title{
ESTRATEGIA COLABORATIVA DE MEJORA DE LA CALIDAD PARA UN EVE-A: EL CASO e-MEM
}

David Rodríguez Gómez.

Universidad Autónoma de Barcelona, España

Lo virtual no es, en modo alguno, lo opuesto a lo real, sino un forma de ser fecunda y potente que favorece los procesos de creación, abre horizontes, cava pozos llenos de sentido bajo la superficialidad de la presencia física inmediata..

Pierre Lévy

\section{A MODO DE INTRODUCCIÓN...}

Durante el último lustro han proliferado enormemente lo que conocemos como LMS $^{1}$ (Learning Management System), $\mathrm{CMS}^{2}$ (Content Management System), o una combinatoria de ambos sistemas, los LCMS (Learning Content Management System).

La necesidad e importancia de promover el uso de las TIC para aumentar las oportunidades educativas y/o formativas está provocando que la mayoría de universidades españolas estén requiriendo la utilización de este tipo de plataformas para poder ofertar formación on-line, sobretodo en tercer ciclo y formación continuada, a las ofertas presenciales ya existentes.

Estos nuevos entornos de formación on-line requieren de una serie de consideraciones y/o aspectos técnicos y pedagógicos que aseguren la calidad de la formación ofrecida.

Sin embargo, el uso de este tipo de plataformas no siempre va acompañado del necesario análisis pedagógico y organizativo que garantizaría una respuesta adecuada y ajustada a las necesidades educativas y/o formativas de la institución solicitante.

Desde el grupo MEM, del Dep. de Pedagogía Aplicada de la Universidad Autónoma de Barcelona, estamos investigando y desarrollando un Entorno Virtual de Enseñanza-Aprendizaje -EVE-A-, concebido especialmente para estudios de postgrado y bajo unos criterios pedagógicos y técnicos que aseguren su calidad como entorno formativo: e-MEM.

Definimos un Entorno Virtual de Enseñanza-Aprendizaje como un espacio de intercambio, de comunicación, de interacción y de encuentro donde, a través del soporte tecnológico, se desarrollan, no solamente, procesos de enseñanza-aprendizaje, sino también procesos de relaciones sociales que enriquecen el proceso formativo, favoreciendo la creación conjunta de conocimiento.

\section{ALGUNAS NOTAS SOBRE LA PLATAFORMA e-MEM}

La plataforma eMEM, escalable y modular, se ha generado a partir del núcleo de programación Php-Nuke, publicado bajo licencia GNU/GPL, con la intención de convertir sus funcionalidades 'open source'

\footnotetext{
${ }^{1}$ Paquete integrado de software, alojado en un servidor, que ayuda a organizar y gestionar acciones formativas on-line.

${ }^{2}$ Herramienta para la gestión de la información que permite su actualización automática cada cierto periodo de tiempo.
} 
en un potente LCMS (Learning Content Management System) con todas las herramientas y características necesarias para diseñar, generar y crear una plataforma de teleformación dinámica potente y de bajo coste.

El siguiente cuadro resume las características técnicas básicas de la plataforma de teleformación eMEM:

Cuadro 1.

Características técnicas de e-MEM ${ }^{3}$

\begin{tabular}{|c|c|}
\hline Nombre de la plataforma & e-MEM \\
\hline Idioma & $\begin{array}{l}\text { Multi-idioma: hasta un total de } 25 \text { (actualmente } \\
\text { únicamente se han comprobado el castellano y el } \\
\text { catalán). } \\
\text { La selección del idioma es personalizable por el } \\
\text { Administrador (para toda la plataforma) o por el } \\
\text { propio usuario. }\end{array}$ \\
\hline Tecnología de programación & PHP+MySQL+FLASH \\
\hline Versión de la Plataforma & 1.2 (estable) \\
\hline Hardware Servidor & $\begin{array}{l}\text { Mono-procesador Pentium 2.4Ghz } \\
1 \text { GB RAM } \\
2 \mathrm{HD} \times 60 \text { GB } 7200 \mathrm{rpm}\end{array}$ \\
\hline Software Servidor & $\begin{array}{l}\text { Sistema operativo LINUX (Debian) } \\
\text { Servidor web Apache 1.3.27 } \\
\text { Servidor de BD MySQL 4.0.12 } \\
\text { MyphpAdmin 2.4.0 } \\
\text { Servidor de mail } \\
\text { Servidor FTP } \\
\text { Servidor Macromedia Flash Comunication } 1.5 \\
\text { Lenguaje PHP Script 4.3.1 } \\
\text { Núcleo Nuke } 6.5+\text { PHPBB Fòrums 2.0.4 }\end{array}$ \\
\hline Red & $\begin{array}{l}\text { Ethernet } 100 \mathrm{Mb} \\
\text { Ancho de banda recomendable mín } 2 \mathrm{~GB}, \mathrm{ATM} \text { u } \\
\text { otros (en función del número de usuarios) }\end{array}$ \\
\hline Distribución & $\begin{array}{l}\text { No distribuible actualmente. Servicio de hosting y } \\
\text { housing vía web }\end{array}$ \\
\hline Licencia & GNU-GPL \\
\hline Actualización & Constante \\
\hline Demo & http://mem.uab.es/ (en pruebas) \\
\hline
\end{tabular}

Continuemos con las principales funcionalidades de la plataforma e-MEM:

Cuadro 2.

Funcionalidades del plataforma e-MEM ${ }^{4}$

\begin{tabular}{|c|c|}
\hline Perfil de usuarios & $\begin{array}{l}\text { Usuario externo (usuario del portal y del centro } \\
\text { de recursos) } \\
\text { del ssistema) } \\
\text { duario interno (coord. del curso, administrador } \\
\text { Usuario formativo-discente } \\
\text { profesor dinamizador, profesor orientador) }\end{array}$ \\
\hline \multicolumn{2}{|c|}{ Areas básicas para usuarios registrados } \\
\hline Area de comunicación & $\begin{array}{ll} & \text { Foros } \\
\text { - } & \text { Correo interno } \\
\text { Chat } & \text { Videochat instantánea } \\
\text { Intranet } \\
\text { Revista de colaboración } \\
\text { Grupos de trabajo }\end{array}$ \\
\hline Area de formación & $\begin{array}{l}\text { Módulos de aprendizaje: } \\
\text { Conceptualización pedagógica (programa, } \\
\text { actividades, glosario, bibliografía, etc.) } \\
\text { Contenidos de aprendizaje en formato } \\
\text { multimedia. }\end{array}$ \\
\hline
\end{tabular}

\footnotetext{
${ }^{3}$ Cuadro adaptado de documentos internos de trabajo del grupo MEM

${ }^{4}$ Cuadro adaptado de documentos internos de trabajo del grupo MEM.
} 


\begin{tabular}{|c|c|}
\hline Area de Recursos & $\begin{array}{ll}\text { - } & \text { FAQ's } \\
\text { - } & \text { Buscador } \\
\text { - } & \text { Zonculos de interés } \\
\text { - } & \text { Galería de recurga } \\
\end{array}$ \\
\hline Area personal & $\begin{array}{ll}\text { - } & \text { Agenda (personal y del curso) } \\
\text { - } & \text { Eatos personales } \\
\text { - } & \text { Evaluacionte académico } \\
\end{array}$ \\
\hline Area de administración & $\begin{array}{ll} & \text { Normativa del curso } \\
& \text { Staff } \\
& \text { Requerimientos técnicos } \\
\end{array}$ \\
\hline \multicolumn{2}{|l|}{ Areas específicas } \\
\hline Profesores y tutores & $\begin{array}{l}\text { - Seguimiento global del módulo de aprendizaje } \\
\text { - } \quad \text { Seguimiento individualizado de los estudiantes } \\
\text { - Sistema de evaluación on -line }\end{array}$ \\
\hline Coordinador & $\begin{array}{l}\text { : } \quad \text { Gestión de cursos } \\
\text { : } \quad \text { Gestión de módulos de contenidos } \\
\text { - Creación de grupos humanos } \\
\text { Asignación de funcionalidades } \\
\end{array}$ \\
\hline Administrador & $\begin{array}{l}\text { - Control total sobre el sistema vía web } \\
\text { - Acceso directo a los recursos del servidor }\end{array}$ \\
\hline
\end{tabular}

Seguramente, no son las características técnicas de eMEM lo que hacen de él un EVE-A de calidad, sino el hecho de que, a diferencia de la mayoría de plataformas y/o EVE-A existentes hasta el momento, e-MEM ha partido de unos principios pedagógicos previos y fundamentadores que han condicionado y guiado su desarrollo tecnológico posterior.

\section{ACERCA DE LA CALIDAD}

La palabra 'calidad' pretende otorgar un sello de garantía y reconocimiento a la realidad a la que se aplica. Calidad es, también, un anhelo, un deseo de perfección, un objetivo al que aproximarse pero que nunca se consigue del todo.

Álvaro Marchesi

A pesar del carácter subjetivo y polisémico del termino 'calidad' no hay duda de que, en la actualidad, se trata de un objetivo deseable y un referente obligado para cualquier organización que se precie.

La falta de una teoría sobre la calidad y las dificultades para poder llegar a un consenso entre las distintas audiencias implicadas impide que se pueda establecer una definición de este concepto que sea aceptada de forma unánime (...).

Lo mismo sucede cuando nos referimos a calidad de la educación. La delimitación de este término al ámbito educativo no ayuda de manera especial a precisar su definición, dado que puede ser igualmente abordado partiendo de diversos enfoques y criterios. (De Miguel, cit. en Gairín, 1999: 13)

La gestión de la calidad es considerada en los ambientes especializados en el estudio de las organizaciones como la estrategia de progreso por excelencia para las próximas décadas.

Se trata de un marco orientador suficientemente amplio, con la categoría a nuestro juicio de paradigma, que incluye valores, principios, normas, planes y procedimientos. (López, cit. en Gairín, 1999; 21)

La estrategia de mejora que presentamos aquí parte de considerar la calidad como la capacidad para responder a las exigencias del entorno.

Inicialmente partimos de un enfoque tecnológico (paradigma científico-racional) que nos llevó a diseñar un modelo de calidad de EVE-A, pero conscientes de sus limitaciones tendimos hacia un enfoque 
más político (paradigma socio-crítico) incluyendo una metodología dinámica y dialéctica que nos permitiera profundizar en el conocimiento de la realidad.

\section{HUYENDO DE LOS MODELOS TECNOCÉNTRICOS DE EVE-A}

El modelo de EVE-A al que hemos llamado tecnocéntrico ha sido el predominante hasta el momento en el ámbito educativo.

Este tipo de modelo se caracteriza, en el mejor de los casos, por una fuerte inversión en tecnología, sin considerar una selección previa de esa tecnología en función de las necesidades educativas del grupo diana. Esto supone que el diseño pedagógico, fundamental en cualquier acción formativa, queda subordinado a esa tecnología.

Cuadro 3.

Modelo tecnocéntrico de EVE-A.

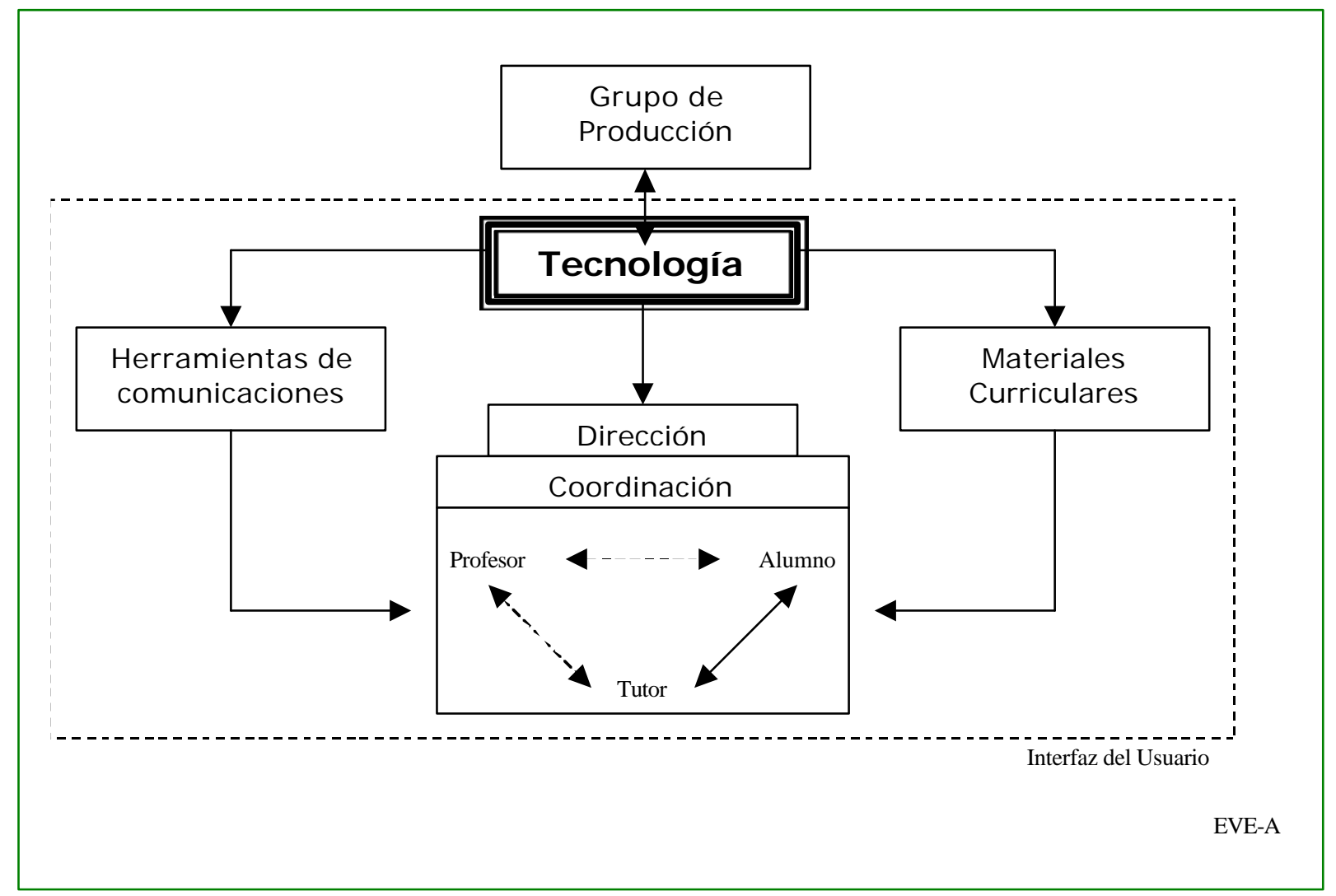

Principales inconvenientes de este tipo de modelos:

- Carencia de una cultura común en todo el entorno;

- Inexistencia de un modelo pedagógico claro;

- Ausencia de una infraestructura tecnológica adecuada;

- Dependencia absoluta de la tecnología y los tecnólogos;

- Como consecuencia de todo ello, puede darse:

- Falta de coherencia entre los objetivos formativos propuestos (en el caso de que existan) y el desarrollo de los diferentes cursos. 
- Baja interrelación entre los miembros del entorno (profesores, tutores, alumnos, diseñadores gráficos, técnicos informáticos, expertos en desarrollo de contenidos, dirección y coordinación);

- Baja interacción de los alumnos con los contenidos, con los profesores y con el resto de alumnos (dimensión cognitiva);

- Baja participación de los alumnos y profesores en el entorno (dimensión social).

\section{PROPUESTA DE UN MODELO DE CALIDAD DE UN EVE-A}

Como podemos observar en el cuadro 4 , el modelo propuesto, contrariamente al modelo tecnocéntrico anterior, se centra en el proceso de enseñanza-aprendizaje a partir del cuál se seleccionarían y/o desarrollarían las tecnologías más apropiadas. Pero no sólo eso, sino que todo el eMEM queda enmarcado en una determinada concepción pedagógica en constante interacción con el contexto sociocultural y económico externo, con la finalidad de responder en todo momento a las necesidades y/o demandas surgidas de ese contexto externo.

Cuadro 4.

Propuesta de un Modelo de Calidad de un EVE-A

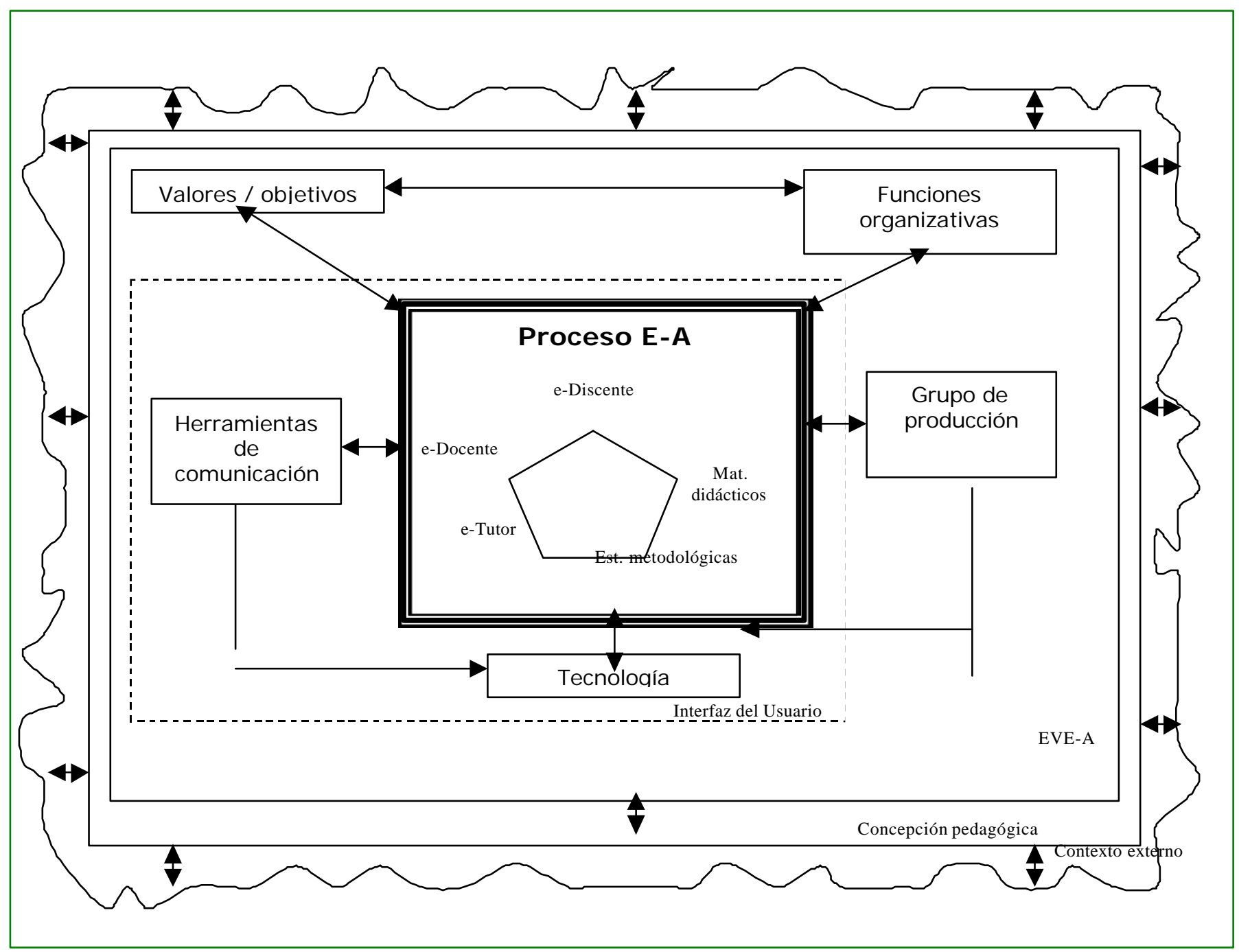


Se tienen en consideración, por tanto, los principales elementos de cualquier acto didáctico y las interrelaciones que entre ellos se producen:

- Contexto: cualquier tipo de relación e interacción se produce en un contexto particular que la determina y condiciona. Así pues, el contexto es un elemento fundamental a tener en cuenta el diseño del modelo psicopedagógico de un EVE-A.

- Estrategias metodológicas: esenciales para optimizar el proceso de enseñanza-aprendizaje. En el caso de la enseñanza virtual se debe considerar aspectos como: medios y recursos, actividades, temporalización, lenguaje, actividades, etc.

- Materia: contenido seleccionado y secuenciado para el proceso formativo en cuestión.

- Usuarios: en un EVE-A los usuarios implicados directamente en el acto didáctico son el profesor/a (experto-autor de contenidos), el alumno/a (construye su aprendizaje) y el tutor/a (guía-referente del alumno durante el proceso de aprendizaje).

\section{ESTRATEGIA COLABORATIVA DE MEJORA DE LA CALIDAD DEL E-MEM}

El correcto desarrollo y utilización de un EVE-A puede aportar enormes beneficios a los procesos de enseñanza-aprendizaje, logrando, por ejemplo, mayores cotas de individualización en los procesos de E-A. Pero como cualquier otro medio, una mala utilización pervierte sus virtudes y comporta una degeneración de los procesos formativos.

Esta es una de la razones que nos ha llevado a desarrollar y llevar a cabo una estrategia de cambio y mejora colaborativa que permita asegurar la calidad tecnológica, educativa y formativa de e-MEM ante los continuos avances tecnológicos de una sociedad con necesidades tan diversas y cambiantes como la nuestra.

Conscientes del peligro de caer en un enfoque mecanicista y predeterminado hemos intentando dotar de una gran flexibilidad a todo el proceso, permitiendo la posibilidad de avanzar y retroceder en sus fases tantas veces como sea necesario, siempre y cuando la evaluación del proceso así lo recomiende.

A lo largo de todo el proceso deben tenerse en cuenta constantemente unos referentes que dan consistencia y sentido a la estrategia: formación, reflexión, negociación, flexibilidad, participación, colaboración y retroalimentación.

\section{POSIBILIDADES Y LÍMITES DE LA ESTRATEGIA DE MEJORA}

Como cualquier modelo, estrategia o plan de mejora, la estrategia aquí propuesta presenta a priori toda una serie de posibilidades y límites de los cuáles ofreceré una pequeña muestra a continuación.

Sin más pretensión que la de dejar un 'buen sabor de boca' empezaré por las limitaciones para así poder finalizar con las posibilidades de la estrategia de mejora para un EVE-A.

Limitaciones de la estrategia de mejora:

- La propia dimensión colaborativa de la estrategia supone una limitación en aquellos casos en los que nos encontremos con un EVE-A con una amplia comunidad, ya que la colaboración / 
participación en el proceso de mejora, en el mejor de los casos, se convertirá en algo simplemente testimonial.

Cuadro 5.

Estrategia colaborativa de mejora de la calidad en un EVE-A

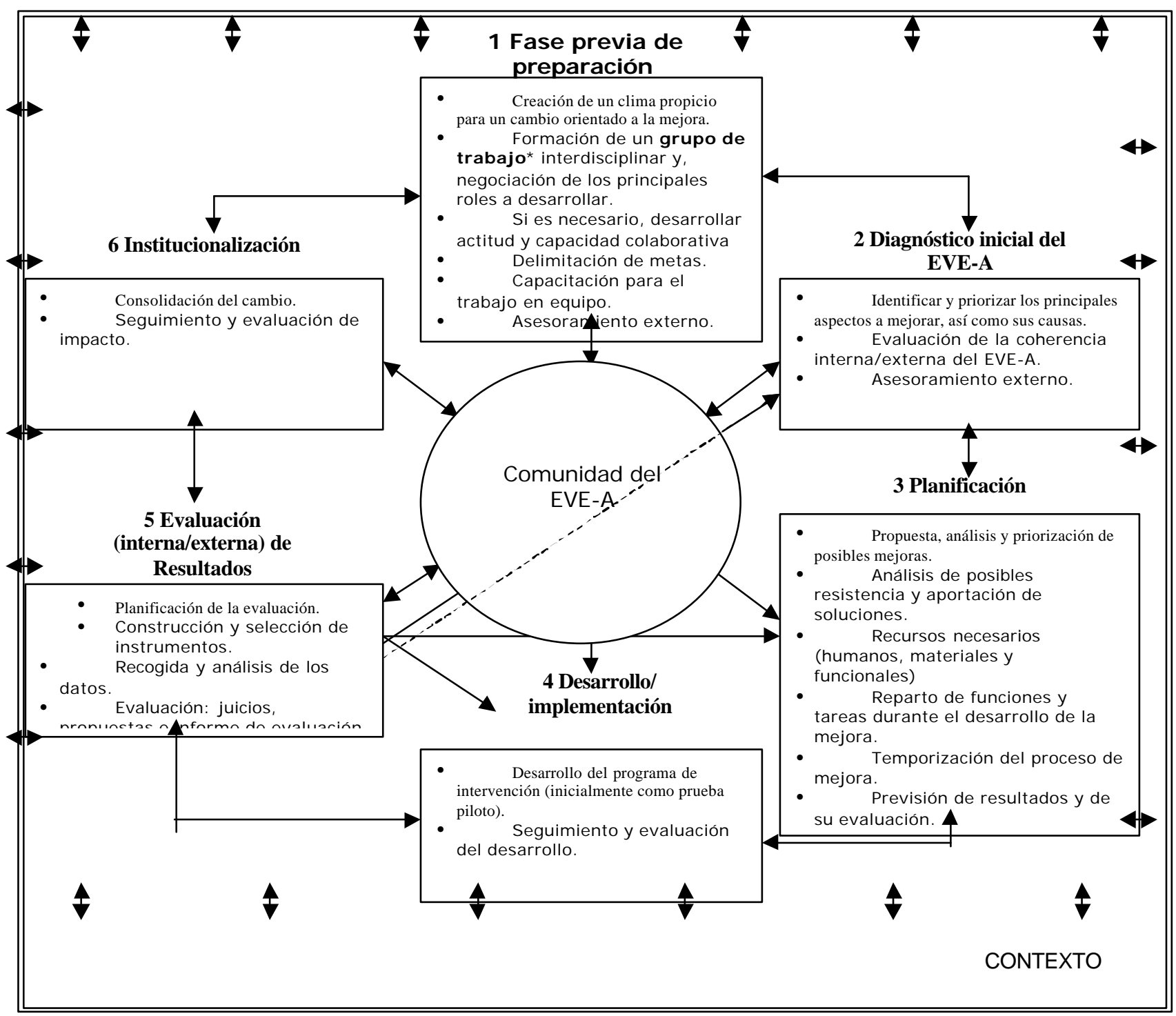

- La continua interacción con el contexto y con el resto de la comunidad del EVE-A puede relantizar exageradamente el proceso de mejora.

- El hecho de que sea un pequeño "Grupo de mejora" el principal encargado de desarrollar toda la estrategia de cambio puede desvincular al resto de miembros de la comunidad del proceso, y generar en ellos la sensación de que se trata de un cambio impuesto desde fuera y, por tanto, provocar la aparición de algunas resistencia a dicho cambio.

- La consolidación del cambio, como en cualquier organización, puede resultar una tarea ardua y complicada que puede requerir de agentes internos o externos que la apoyen, pero esto a la vez puede generar una dependencia hacia esos agentes. 
- Posibilidades de la estrategia de mejora:

- Al no tratarse de una estrategia de mejora lineal y cerrada, le proporciona una gran flexibilidad y dinamismo que le permite rápidamente adaptarse a los cambios y enmendar cualquier error producido durante el proceso de mejora.

- A pesar de tratarse de una estrategia de mejora pensada expresamente para eMEM, resulta fácilmente adaptable a cualquier otro tipo de EVE-A y/u organización.

- El carácter colaborativo de la estrategia proporciona a todo el proceso una mayor aceptación por parte de todos los miembros de la comunidad y disminuye las posibles resistencias que pudieran presentarse.

\section{BIBLIOGRAFÍA}

ADELL, J. Tendencias en educación en la sociedad de las tecnologías de la información. EDUTEC, núm. 7 (noviembre, 1997). URL: http://www.uib.es/depart/gte/revelec7.html

AREA MOREIRA, M. La tecnología educativa y el desarrollo e innovación del currículum. Ponencia presentada en el XI Congreso Nacional de Pedagogía. San Sebastián, 1996.

AREA MOREIRA, M. Futuro imperfecto. Nuevas tecnologías y desigualdades educativas. Ponencia presentada en XX Escuela de Verano de Canarias. Islas Canarias, 1997. URL: http://www.ull.es/publicaciones/tecinfedu/NT1.htm

AREA MOREIRA, M. (coord.) Educar en la sociedad de la información. Bilbao: Descleé de Brouwer, 2001.

AREA MOREIRA, M. Igualdad de oportunidades y nuevas tecnologías. Un modelo educativo para la alfabetización tecnológica. EDUCAR, núm. 29 (2002): 55-65.

BARTOlomé, A. Preparando un nuevo modo de conocer. EDUTEC, núm. 4. (diciembre, 1996). URL: http://www.uib.es/depart/gte/revelec4.html

BATES, A.W. Cómo gestionar el cambio tecnológico. Gedisa-EDIUOC: Barcelona, 2001.

CABERO, J. Nuevas tecnologías, comunicación y educación. EDUCTEC, núm. 1. (febrero, 1996). URL: http://www.uib.es/depart/gte/revelec.1.html

CASTELLS, M. La era de la información. Economía, sociedad y cultura. Vol. 2. Alianza: Madrid, 1998.

CASTEllS, M. La Galaxia Internet. Plaza Janés: Noviembre, 2001.

CASTELLS, M. Internet, libertad y sociedad: una perspectiva analítica. Lección inaugural del curso académico 2001-2002 de la UOC. Barcelona: UOC, 2001. URL: http://www.uoc.edu/web/esp/launiversidad/inaugural01/intro conc imp.hml

Colom, A.; SARRAmONA, J.; VÁZQUEZ, G. Estrategias de formación en la empresa. Narcea: Madrid, 1994.

COMISIÓN EUROPEA. Libro blanco sobre la educación y la formación. Enseñar y aprender. Hacia la sociedad del conocimiento. Luxemburgo: Oficina de Publicaciones Oficiales de las Comunidades Europeas, 1995.

DELORS, J. La educación encierra un tesoro. Santilla-UNESCO: Madrid, 1996. 
Foro De LA SOCIEDAD DE LA INFORMACIÓN. Redes al servicio de las personas y las colectividades. Cómo sacar el mayor partido de la sociedad de la información en la Unión Europea. Primer informe anual del Foro de la Sociedad de la Información a la Comisión Europea. URL: http://www.ispo.cec.be/infoforum/pub.html

DORADO; C.; RodríGueZ, D., Un enfoque de entorno virtual de aprendizaje centrado en los usuarios. En soporte CD, del IV Congreso Internacional Virtual de Educación - CIVE 2004.Palma de Mallorca.

GAIRÍN, J.; DARDER, P. Organización de centros educativos. Praxis: Barcelona, 1994.

GaIRín, J. La organización escolar: contexto y texto de actuación. La muralla: Madrid, 1996.

GAIRín, J. La calidad, un concepto controvertido. Educar, núm. 24. UAB: Bellaterra, 1999.

Gonzalez Soto, A.P. et alt. Las nuevas tecnologías en la educación. En Salinas et. Alt. Redes de comunicación, redes de aprendizaje. EDUCTEC'95. Palma: Universitat de les Illes Balears: 409-422. URL: http://www.uib.es/depart/gte/grurehidi.html

HANNA, D. La enseñanza universitaria en la era digital. Octaedro-EUB: Barcelona, 2002.

LÉVY, P. ¿Qué es lo virtual?. Paidós: Barcelona, 1999.

MAJÓ, J.; Marqués, P. La revolución educativa en la era Internet. Praxis: Barcelona, 2002.

MARCHES, A.; Martín, E. Calidad de la enseñanza en tiempos de cambio. Alianza: Madrid, 1998.

NEGROPONTE, N. El mundo digital. Barcelona: Ediciones B, 1995.

Perelman L.J. (s.f.) The future of technology in Education: A "Multimedia Today" Roundtable Discussion. URL: http://www.multimedia.hosting.ibm.com/mmtoday/magazine/round-1.html

SANCHO, J.M.; Millan, L.M. (coord.) Hoy ya es mañana, Tecnologías y educación: un diálogo necesario. Sevilla: Publicaciones del Movimiento Cooperativo de la Escuela Popular, 1995.

SIMONE, R. La Tercera Fase. Formas de saber que estamos perdiendo Madrid: Taurus, 2001.

VVAA. Educar para la sociedad de la información. Nuevas necesidades, viejas estructuras. Telos. Cuadernos de Comunicación, Tecnología y Sociedad. URL:

WOLTON, D. Internet ¿Y después qué?. Barcelona: Gedisa, 2000.

Zubero, I. Participación y democracia ante las nuevas tecnologías. Retos políticos de la sociedad de la información. Telos. Cuadernos de Comunicación, Tecnología y Sociedad. URL: 


\title{
Contactar
}

Revista lberoamericana de Educación

\author{
Principal OEI
}

